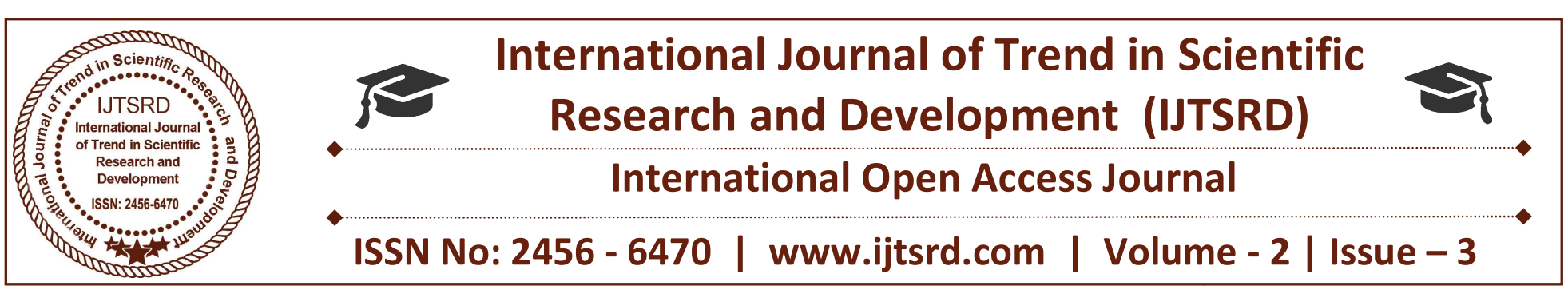

\title{
The Dependency of the Internet of Things on Cloud Computing
}

\author{
Mohammad Asim \\ Jamia hamdard, New Delhi, India
}

\author{
Ranjit Biswas \\ Jamia hamdard, New Delhi, India
}

\begin{abstract}
Cloud and IoT two out and out various developments are both starting at now part of our life. An extraordinary worldview region Cloud and IoT are circumscribed is anticipated as alarming, what's more, an empowering specialist of a broad number of employment circumstances. This paper focuses on the blend of Cloud and IoT, which is denoted by the Cloud IoT perspective. Various works in composing have reviewed Cloud and IoT freely: their fundamental virtue, focal points, hidden growth, and problems. Be that as it may, to the best of our insight, these works do not have a point by point investigation of the Cloud IoT worldview. To connect this hole, we audit the writing about the coordination of It and cloud. We begin dissecting and examining the requirement for incorporating them, the difficulties getting from such reconciliation, and how these issues have been handled in writing. We by then depict application circumstances that have been shown in composing, and what's more stages both business and publicly accessible and ventures executing the Cloud IoT perspective. At long last, we determine open issues, precept problems and destiny bearings in this promising discipline.
\end{abstract}

Keywords: IoT, Distributed computing, CloudIoT worldview

\section{INTRODUCTION}

The Internet of Things refers to the ever-developing community of bodily gadgets that functions an IP address to connect to the internet and the verbal exchange that happens between these items and different Internet-enabled gadgets and systems. IoT is by and large described by true and little things with restricted capacity, handling limit, and significant issues with respect to unwavering quality, execution, safety, and protection. Whereas, Cloud computing acquire essentially limitless abilities on par with capacity and preparing power, is notably more develop innovation, and has the vast majority of the IoT issues in any event incompletely settled. In this manner, a novel IT worldview in which Cloud and IoT are two reciprocal innovations consolidated is the norm to upset both present and future Internet. This paper surveys the writing about the combination of Cloud and IoT an extremely encouraging subject for both research and industry.

We at first give a common depiction of the composition going for showing up subjectively the transient direct of the examination and the essential energy about the CloudIoT perspective. Secondly, we give a definite dialog on the IoT and cloud, featuring the requirement for their incorporation. Thirdly, we element the brand new utility conditions coming from the choice of the CloudIoT worldview. Fourth, together with breaking down the CloudIoT worldview and the software conditions, we infer the new points and associated troubles for inquiring about. Then, we painting the fundamental stages (commercial enterprise and open source) and look into ventures in the discipline of CloudIoT. At last, we discuss the problems and future scope in the knowledge domain of cloudIoT.

\section{LITERATURE REVIEW}

Internet of Things (IoT) term addresses a general thought for the limit of framework contraptions to differentiate and accumulate information from around 
the world and afterward supply that information over the web whereverit is taken care of andutilizedfor numerous charming functions. Numbers of People have featured the work done on IoT and its applications throughout the years. This segment centers on a writing audit in these zones.

Juan Felipe Corso arias from Granada University displays the outline of a remote correspondence framework, where the sensor idea linked to the mechanical mechanism, which senses the temperature. We can access those sensors from many places as they are NOT based. The data is cached in the cloud repository. PLC is used that interact with sensors. The watching system becomes finished with a SCADA framework and the showing of the correspondence framework changed into carried out making use of the formalism of Petra nets, as a framework that reacts as far as discrete activities. [3] Chirag M Shah et al [1] present an examination on the best way to change the current access control frameworks when gadgets are getting more brilliant day by day. This method of improving the doorway manage framework which guarantees that the schema is far-flung in this manner reducing wiring problem. These data resources are organized in the controller (TM4C123GXL-in light of ARM Cortex-M4).Using AI, computations are executed to display and look into amassed records.

Vishwajeet H Bhide, K.J college of Engg., Pune. In this paper, he discloses how to give completely savvy condition by observing by different sensors (Temperature, Humidity, Light, and Level) for giving fundamental information to naturally change the solace level in homes by enhancingutilization of vitality. We likewise utilize forecast here for consequently location and determination of any issue in the gadgets. For that, we are utilizing Naive Bayes Classifier calculation for information mining. It will send email or SMS to a required specialist for administration and it will likewise inform the proprietor. This gives an enormous preferred standpoint on the keen home frameworks utilizing IoT. [15]

Byung Mun Lee* and Jinsong Ouyang, Gachon University, Korea - clarifies that supervision of incessant sicknesses is imperative to own administration for well-being. The IoT idea assumes a noteworthy part of self-administration for well-being. With a specific end goal to achieve it, individual well- being gadgets require two capacities, for example, application organizes the convention and savvy benefit. Be that as it may, the vast majority of them have the just basic capacity, for example, showing estimated information and putting away information incidentally. In this exploration, we proposed a smart administration display for social insurance which gives a compelling criticism to a person. So as to do this, we presented the cooperation convention which exchanges hazard factors between IoT individual well-being gadgets. At long last, in light of the discoveries of the analysis, the adequacy was affirmed on proposed demonstrate. [5]

Chang-Su Ryu et al [4] proposes an Internet of Things (IoT)- based savvy fire crisis reaction framework that can control directional direction brilliantly as per the time and area of a calamity and the outline of a coordinated control framework utilizing remote sensor systems to address the issues with existing fire crisis reaction frameworks in the midst of flame or building breakdown.

P.Elanthiraiyan, Dr. S.Babu, IEFT College of engineering. This paper clarifies how IoT is excelling in the medical sector. One of the better ways the specialists are fit to surely and rapidly ideal to utilize the significant patient data's and including the patient restorative history. Through IoT, enormously enhances the quality of data and howa patient's taken care of in the Medical field. Thus, Internet of Things offers a real stage to interconnect the every one of the assets Ontology helps PCs in furthermore understanding the side effects and therapeutic assets. Along these lines, Ontology makes a recovery system and reconfigures therapeutic assets as indicated by patient's particular prerequisites quickly and over and over. [2]

Kostas E Psanniset al [16]proposed dedication of Cloud to the evolution of IoT. Hence, it demonstrates how Cloud technology modernization complements the ability of the IoT and overviews the safety problems of cloudIoT.

Manual De Buenaga et al [11] portrays the preparatory approach of "Virtual Cloud Carer",Whose simple function is the formation of recent health administrations forwards, utilizing improvements based on smart devices. 
Rodger Lea et al [12] present the general idea of IoT center points and after that talk about our work,to sum up, our IoT center point as a Smart City PaaS. We quickly portray our approach and talk about our encounters sending cloud-based city centers, located in Canada and united kingdom

Subhadeep Sarkar et al [13] clarifies near examination of the mist figuring worldview and the ordinary distributed computing worldview with regards to smart devices, by scientifically defining the parameters and qualities of mist registering - one of the primary endeavors of its kind. With the quick increment in the quantity of Internet-associated gadgets, the expanded interest for constant, lowinactivity administrations is turned out to be trying for the customary distributed computing system.

\section{COLLABORATING IOT AND CLOUD}

The two universes of Cloud and IoT have seen a free development. Be that as it may, a few common focal points getting from their reconciliation has been recognized in writing and is anticipated later on. From one perspective, IoT benefits for all intents and limitless capability and belongings of Cloud to modify its modern necessities (e.g., efficiency, preparing, and vitality). IoT makes use of numerous cloud functionalities like stashing and evaluate the info to abbreviate its professional hindrance. Likewise, cloud exploits IoT by dealing with the info in an indispensable way and yield answers to genuine issues.Next, we compile the issues that have been addressed and the benefits of CloudIoT worldview.

Data repository: IoT includes an expansive sum of data from various sources, which deliver a colossal a measure of unorganized information focuses on $3 \mathrm{~V}$ 's that are volume (information measure), assortment (information writes), and speed (information age recurrence). Consequently, it suggests gathering, getting to, and handling, envisioning, documenting, sharing, and seeking vast measures of information. Offering basically boundless, minimal effort, what's more, on-request stockpiling limit, Cloud is the most helpful what's more, the savvy answer for management information delivered by IoT. [17]

Communication Resources: One of the necessities of IoT is to make IP-empowered gadgets convey through devoted equipment and the help for such correspondence can be exceptionally costly. Cloud offers a viable and modest arrangement to interface, track, and oversee anything from anyplace at whenever utilizing altered entries and implicit applications. Because of the accessibility of rapid systems, it empowers the checking and controlling remote devices, what's more, the ongoing access to the delivered information

State-Of-The-Art: The selection of the CloudIoT worldview empowers new situations for shrewd administrations and applications in view of the augmentation of Cloud through the things.

-SaaS programs provide unprecedented flexibility and ease of use;

-SensaSempowering universal administration of remote sensors;

-DBaaS empowering universal directory administration;

- DaaS allows users to access information;

- EaaS gives remote gadgets layer-2 availability; - VSaaS Giving generic access to recorded video and actualizing complicated investigations within the Cloud. [18]

\section{APPLICATIONS}

We are utilizing IoT in our day to day life.It is turning into an essential piece of our quick moving world. Let us discuss few of them.

Smart cities: The term 'Smart Cities' is utilized to indicate the cyber-physical eco-framework developing by sending propelled correspondence foundation and novel administrations over far-reaching situations. By methods for cutting-edge administrations, it is, in reality, conceivable to enhance the utilization of physical city foundations (e.g., street systems, control lattice, and so forth.) and personal satisfaction for its residents. Io $T$ innovations can locate various differing application in savvy urban areas situations. As a contextual investigation, IoT advancements can be used to give propelled activity control frameworks. In this point of view, autos will be comprehended as speaking to 'shrewd objects'. Likewise, brilliant stopping gadgets framework, in view of RFID and sensor innovations, may permit to screen accessible parking spots and give drivers with robotized stopping guidance, along these lines enhancing portability in the urban territory. Additionally, sensors can sense the movement of the vehicles on roadways and collect data, such as speed and quantities of vehicles. Sensors can identify the contamination level of air, recovering brown haze data, for example, the level of carbon 
dioxide, PM10, and so on. And convey such data to wellbeing offices.[12]

Healthcare: IoT innovations can locate various applications in the human services segment.From a point of view, they can be utilized to enlarge current helped lodging courses of the plan. Patients will convey restorative sensors to screen parameters, for example, body temperature, circulatory strain, relaxing action. People will pass across sensors that analyze people's health w.r.t certain parameters. Sensors gather info to analyze silent exercises in their life situations. Information is secretly accumulated also, transferred to distant helpful concentrations, which is then analyzed and stored for fast reaction activities when required.The interconnection of such heterogeneous sensors could give a thorough picture of wellbeing parameters, in this way setting off an intercession by the medicinal staff endless supply of conditions that may prompt wellbeing crumbling, consequently acknowledging preventive care. Another important application division identifies with customized human services and prosperity arrangements. The use of hand-held sensors, together with the correct applications that work with custom devices, involves humans keeping track of day to day activities (walking paths, work is done, etc.), providing recommendations to improve their lifestyle. [11]

Security and surveillance: Security reconnaissance has to turn into a need for big business structures, shopping centers, processing plant floors, auto parks and numerous other open places. Country security situations confront additionally comparative dangers, yet on an alternate scale. IoT-empowered advancements can be utilized to enormously upgrade the execution of current arrangements, giving less expensive and less intrusive contrasting options to the across the board sending of cameras while in the meantime protecting clients' security. Encompassing sensors can be utilized to screen the nearness of hazardous chemicals. Sensors checking the conduct of individuals might be utilized to survey the nearness of individuals acting suspiciously. Proficient early cautioning frameworks can, thusly, be fabricated. Individual recognizable proof by methods for RFID or comparative innovations is additionally an alternative. In any case, in numerous nations, client affiliations are furiously challenging about the security encroachment that could come about because of the across the board appropriation of such an innovation.
At the point when utilized as a part of conjunction with part based get to control frameworks, IoT advances can give the abnormal state of adaptability, having the capacity to adapt to get to approaches (e.g., to various territories of structures) which may change after some time because of calculated changes and additionally to changes in the part of the client and additionally as indicated by relevant data (e.g., a few territories not available on a given day because of redesign works going on). Likewise in this market, the preferences are regarding the upgraded usefulness, better client acknowledgment through the decrease in the utilization of cameras, decreased operational expenses and expanded adaptability in a changing domain. [14]

Smart Home: IoT has extensive application in home situations, where heterogeneous implanted gadgets empower the computerization of regular in-house exercises. With a specific end goal to let an assortment of autonomous single-family keen homes get to reusable benefits over the Internet, the subsequent arrangement ought to fulfill three essential prerequisites: interior system interconnection (i.e., each computerized apparatus in shrewd home ought to have the capacity to interconnect with some other), insightful tremote control (i.e., machines and administrations in the brilliant home ought to be cleverly reasonable by any gadget from anyplace), furthermore, home automation (i.e., interconnected machines inside the home should execute their capacities by means of connecting to administrations given by keen home situated Cloud). A few keen home applications proposed in writing include (remote) sensor systems and execute keen metering answers forgive acknowledgment of machines, keen administration of vitality utilization, lighting, warming, and aerating and cooling. [15]

\section{CHALLENGES IN CLOUDIOT WORLDVIEW}

Security: Cloud-based IoT makes it conceivable to transport information from this present reality to the Cloud. Without a doubt, one especially essential issues which have not yet been settled is the way to give proper authorization principles and approaches while guaranteeing that exclusive approved clients approach the touchy information; this is vital with regards to protecting clients' security, and especially when information trustworthiness must be guaranteed. What's more, when basic IoT applications move into the Cloud, issues emerge in light of the absence of trust in the specialist organization, data with respect to 
benefit level understandings (SLAs), and the physical area of information Sensitive data spillage can likewise happen due to the multi-tenure. In addition, open key cryptography can't be connected to all layers on account of the preparing power requirements forced by IoT objects. New difficulties likewise require particular consideration; for instance, the dispersed framework is presented to a number of conceivable assaults, for example, SQL infusion, session riding, cross-site scripting, and side-channel. Besides, critical vulnerabilities, including session seizing and virtual machine escape are additionally risky.[14]

Heterogeneity: One especially vital test looked at the Cloud-based IoT approach is identified with the broad heterogeneity of gadgets, stages, working frameworks, and administrations that exist and may be utilized for new or created applications. Cloud stages experience the ill effects of heterogeneity issues; for example, Cloud benefits, by and large, accompany restrictive interfaces, hence taking into consideration asset mix in view of particular suppliers. Furthermore, the heterogeneity test can be exacerbated when end-clients receive multi-Cloud methodologies, and therefore administrations will rely upon numerous suppliers to enhance application execution and flexibility.

Big Data: With numerous foreseeing that Big Data will achieve 50 billion IoT gadgets by 2020 , it is essential to give careful consideration to the transportation, access, stockpiling and preparing of the huge measure of information which will be created. Undoubtedly, given late / mechanical advancements, plainly the IoT will be one of the center wellsprings of huge information, and that the Cloud can encourage the capacity of this information for a drawn-out stretch of time, notwithstanding subjecting it with a complex examination. Taking care of the gigantic measure of information delivered is a huge issue, as the application's entire execution is vigorously dependent on the properties of this information administration benefit. Finding an immaculate information administration arrangement which will enable the Cloud to oversee huge measures of information is as yet a major issue. Besides, information trustworthiness is an indispensable component, not just as a result of its impact on the administration's quality, yet in addition in light of security and protection issues, the dominant part of which identify with the outsourced information. [17]
Performance: Exchanging the immense measure of information made from IoT gadgets to the Cloud requires high data transmission. Thus, the key issue is getting sufficient system execution so as to exchange information to Cloud conditions; without a doubt, this is on the grounds that broadband development isn't keeping pace with capacity and calculation advancement. In various situations, administrations and information arrangement ought to be accomplished with high reactivity. [7]

Monitoring: Observing is an essential activity in Cloud Computing with regards to execution, overseeing assets, scope quantification, security, SLAs, and for investigating. Therefore, the Cloudbased IoT approach acquires similar checking requests from the Cloud, despite the fact that there are still some related difficulties that are affected by speed, volume, and assortment attributes of the IoT. [9]

\section{OPEN ISSUES AND FUTURE SCOPE}

This area will address a portion of the open issues and future research headings identified with Cloud-based IoT, which still require more research endeavors. These issues include:

The Demand for Standards: Numerous investigations have featured the issues of the absence of norms, which is viewed as basic in connection to the Cloudbased IoT worldview. In spite of the fact that variously proposed institutionalizations have been advanced by the logical society for the arrangement of IoT and Cloud approaches, clearly models, standard conventions, and APIs are required to take into account interconnection between heterogeneous shrewd things and the age of new administrations, which make up the Cloud-based IoT worldview.

Energy productivity: Late Cloud-based IoT applications incorporate successive information that is transmitted from IoT items to the Cloud, which rapidly expands the hub vitality. Accordingly, creating effective vitality with regards to information preparing and transmission remains a huge open issue. A few headings have been proposed to conquer this issue, for example, pressure advancements, productive information transmission; and information storing procedures for reusing gathered information with the time-inhumane application.

Fog Computing: Fog processing is a model which stretches out Cloud figuring administrations to the 
edge of the system. Like the Cloud, Fog supply conveys application administrations to clients. Haze can basically be viewed as an expansion of Cloud Computing which goes about as a transitional between the edge of the system and the Cloud; without a doubt, it works with inactivity touchy applications that require different hubs to fulfill their postpone necessities. In spite of the fact that capacity, registering, and organizing are the fundamental assets of both Fog and the Cloud, the Fog has certain highlights, for example, area mindfulness and edge area that give geological circulation, and low idleness; additionally, there are a substantial hubs; this is conversely with the Cloud, which is upheld by constant collaboration and versatility.[13]

Cloud Capabilities: As in any arranged condition, security is thought to be one of the principal issues of the Cloud-based IoT worldview. There are more odds of assaults on both the IoT and the Cloud side. In the IoT setting, information honesty, secrecy, and genuineness can be ensured by encryption. Notwithstanding, insider assaults can't be settled and it is likewise difficult to utilize the IoT on gadgets with constrained capacities.

Big Data: In the past area, we examined Big Data as a basic test that is firmly combined with the Cloudbased IoT worldview. In spite of the fact that various commitments have been proposed, Big Data is as yet thought about a basic open issue, and one needs more research. The Cloud-based IoT approach includes the administration and handling of tremendous measures of information coming from different areas and from heterogeneous sources; for sure, in the Cloud-based IoT, numerous applications require entangled errands to be performed continuously. [17]

Security and Privacy: In spite of the fact that security and privacy are both basic research issues which have gotten a lot of consideration, they are as yet open issues which require more endeavors. To be sure, adjusting to various dangers from programmers is as yet an issue. Also, another issue is giving the fitting approval principles and arrangements while guaranteeing that exclusive approved clients approach touchy information; this is vital for protecting clients' security, particularly when information uprightness must be ensured.[14]

\section{CONCLUSION}

The IoT is turning into an inexorably universal registering administration which requires immense volumes of information stockpiling and handling capacities. The IoT has restricted abilities as far as preparing force and capacity, while there additionally exist considerable issues, for example, security, protection, execution, and dependability; As such, the joining of the Cloud into the IoT is extremely advantageous regarding conquering these difficulties. In this paper, we exhibited the requirement for the production of the Cloud-based IoT approach. A discourse likewise centered on the Cloud-based IoT design, diverse applications situations, challenges confronting the effective mix, and open research headings. In future work, various contextual analyses will be completed to test the adequacy of the Cloudbased IoT approach in social insurance applications.

\section{REFERENCES}

1. 1.Chirag M. Shah, Vamil B. Sangoi, and Raj M. Visharia, "Smart Security Solutions based on Internet of Things (IoT)", International Journal of Current Engineering and Technology, 01 Oct 2014, thllVol.4, No.5 (Oct 2014) http://inpressco.com/wpcontent/uploads/2014/09/Paper573401-3404.pdf

2. P.Elanthiraiyan, Dr. S.Babu," Smart Medicine and Physical Health System Using IoT', International Journal of Computer Science and Mobile Computing, Vol.4 Issue.3, March- 2015, pg. 333338

3. Juan Felipe Corso Arias,' 'Wireless Sensor System According to the Concept of IoT -Internet of Things-', International Journal of Advanced Computer Science and Information Technology (IJACSIT) Vol. 3, 2014, Page: 327-343, ISSN: 2296-1739

4. Chang-Su Ryu, Department of Cartoon \& Game Animation, Yewon Arts University, Seoul 482863, Korea, "IoT-based Intelligent for Fire Emergency Response Systems", International Journal of Smart Home Vol. 9, No. 3 (2015)

5. Byung Mun Lee* and Jinsong Ouyang," Intelligent Healthcare Service by using Collaborations between IoT Personal Health Devices", International Journal of Bio-Science and Bio-Technology Vol.6, No.1 (2014), pp.155164

6. N. Bergmann andL.Hou,"Novel industrial wireless sensor networks for machine condition 
monitoring and fault diagnosis," Instrumentation and Measurement, IEEE Transactions on, vol. 61, no. 10, 2012.

7. X. Wang-hui, S. Heejong, Z. Ke, and L. Yang, "The application of a wireless sensor network design based on ZigBee in petrochemical industry field," in Intelligent Networks and Intelligent Systems,2008. ICINIS '08. First International Conference on, 2008, pp. 284-287.[4] G. Cena, A. Valenzano, and S. Vitturi, "Wireless extensions of wired industrial communications networks," in Industrial Informatics, 2007 5th IEEE International Conference on, vol. 1, 2007

8. J. CERVANTES, "Representation y aprendizaje de Conocimiento con rides de Petri defuses," 2005.[15] Corso, F.; Camargo, Y.; Ramirez, L., "Wireless Sensor System According to the Concept of IoT -Internet of Things-," Computational Science and Computational Intelligence (CSCI), 2014 International Conference on, vol.1, no., 10-13 March 2014

9. J. Barros, R. Pais, L. Gomes, A. Costa, T. Rodrigues, and R. Ferreira, "Petri net based building automation and monitoring system," in Industrial Informatics, 2007 5th IEEE International Conference on, vol. 1, 2007.

10. Z. Yu, and G. Fu, X. Fu, and Z. Ma, "On Wireless sensor networks formal modeling based on Petri nets," in Wireless Communications, Networking and Mobile Computing (WiCOM), 20117 th International.

11. http://ieeexplore.ieee.org/abstract/document/6296 977/

12. http://ieeexplore.ieee.org/abstract/document/7037 764/

13. http://ieeexplore.ieee.org/abstract/document/7286 $781 /$

14. https://arxiv.org/abs/1501.02211

15. http://www.ijarcsms.com/docs/paper/volume2/issu e12/V2I12-0005.pdf

16. https://www.researchgate.net/publication/3113380 93_Secure_integration_of_IoT_and_Cloud_Comp uting

17. https://www.researchgate.net/profile/Samuel_Foss o_Wamba/publication/265702116_Research_Dire ctions_on_the_Adoption_Usage_and_Impact_of_t he_Internet_of_Things_through_the _Use_of_Big Data_Analytics/links/54197be30cf25ebee $98861 \mathrm{c} 9$ .pdf

18. P.Parwekar,"From the Internet of things to the cloud of Things," Int. Conf. Comput. Commun. Technol., 2011, pp. 329-333. 